\title{
Standard Description of Common Element Model Data Type Based On XML Schema
}

\author{
Yang Qi, Wang Zhulin, Wang Hongyun \\ Department of Missile Engineering, Army Engineering University, Shijiazhuang, 050003, China.
}

\begin{abstract}
AI-ESTATE standard uses EXPRESS language to describe its diagnostic knowledge, but EXPRESS is not a programming language, which makes it difficult to implement diagnostics using EXPRESS language description. This makes it difficult to share and reuse diagnostic knowledge; XML language with its good flexibility, readability and extensibility, it has brought great convenience to information exchange. Mapping the EXPRESS language to XML Schema is of great significance for the sharing of diagnostic knowledge. First, the data types of the EXPRESS language and XML Schema are analysed; then, mapping mechanism of the simple data types, aggregate data type, and structural data types to XML data types in EXPRESS are studied separately. Finally, based on the analysis of the AI-ESTATE standard common element model data type, the process of describing the diagnostic knowledge of the common element model using XML Schema standardization is studied, and the diagnostic knowledge can be

portable

and

reused.
\end{abstract}

\section{INTRODUCTION}

With the increase in the performance and complexity of weapons and equipment, traditional test and diagnostic techniques and ideas have exposed a large number of problems. At this stage, weapons and equipment testing and diagnostic systems are mostly aimed at a specific one or a certain type of equipment. There is a general lack of versatility, scalability, and poor portability, and it is difficult to achieve the sharing and reuse of diagnostic knowledge and data. Not only caused a lot of waste of resources, but also hindered the development of fault diagnosis technology.AI-ESTATE standard regulate test environment under the intelligent diagnosis of knowledge, data and service description, in order to achieve diagnostic knowledge can be exchanges, reuse and shared provide a way ${ }^{[1]}$. This standard contains 5 kinds of diagnosis model ${ }^{[2-3]}$, of which the Common Element Model (CEM) is the foundation model, others. Models are extended on the basis of CEM, the model uses EXPRESS language to describe the diagnostic knowledge, as EXPRESS language can not be directly used to achieve programming, making diagnosis difficult to share. Under this circumstance, this article seeks to use the diagnostic model defined by the AI-ESTATE standard as a basis, to seek a language that is more advantageous in terms of data exchange to standardize the information description, and the XML language is undoubtedly a good choice. Based on AI-ESTATE, a standardized description of the diagnostic model can be implemented based on XML Schema ${ }^{[4]}$.

\section{XML SCHEMA AND EXPRESS}

\section{$2.1 \quad \mathrm{XML}$ Schema}

This article uses XML Schema to define the document structure. XML Schema divides data into two main categories: simple type and complex type. Containing attributes or other elements are complex type, and this article uses XML Schema to define the document structure. XML Schema divides data into two main categories: simple type and complex type. Containing attributes or other elements are complex type, simple type include only attribute values and textual content. Simple type have 3 main derived type: restriction type, form type and union type. The most common of these is the restriction type, defined by <restriction> declaration. one of simple type unique characteristics is the "Constraints Face", XML Schema have 12 constraints surface, by defining each constraint surface to achieve about the data bunching and control ${ }^{[5]}$.

\subsection{EXPRESS}

EXPRESS is a canonical information modelling language that provides a detailed description of the specification of objects in the ontology and provides a description of the information and constraints associated with those objects. EXPRESS language is not a programming language, does not contain input and output, information processing 
and management of language elements, is not conducive to the model processing work. EXPRESS data type can be divided into: simple data type, aggregate data type, named data type, structural data type. The type of composition is as follows:

- Simple data type. NUMBER, INTEGER, REAL, LOGICAL, BOOLEAN, BINARY, STRING.

- Aggregate data type. ARRAY, BAG, LIS, SET.

- Named data type. Entity type, Defined Type.

- Structural data type. Enumeration type, Select Type.

\section{MAPPING OF EXPRESS TO XML SCHEMA}

EXPRESS data type include simple data type, aggregate type, named data type, structural data type, which named data type consists of entity type and Defined type, these two kinds of data type composition is more complex, may include simple data type, aggregate data type and structural data type in one or several. So the mapping these data type is the basis of type mapping.

\subsection{Mapping of constant}

A constant type CONSTANT can be described in EXPRESS, but the constants are not defined in the XML, so CONSTANT EXPRESS description does not exactly match the XML description. We can only follow the principle of name correspondence, meaning and consistency. A constant description is converted to an element, the constant identifier is converted to the element's name attribute, the base type is converted to an EXPRESS element attribute, the expression is a child element, and the XML of the EXPRESS constant is described as follows:

$$
\begin{aligned}
& \text { <xs:element name="CONSTANT"> } \\
& \text { <xs:complexType> } \\
& \text { <xs:sequence> } \\
& \text { <xs:element ref="doc:description" minOccurs="0"/> } \\
& \text { <xs:group ref="doc:datatype"/> } \\
& \text { </xs:sequence> } \\
& \text { <xs:attribute name="name" type="doc:identifier" } \\
& \text { use="required" /> } \\
& \text { <xs:attribute } \\
& \text { type="doc:express.text" use="required"/> } \\
& \text { </xs:complexType> } \\
& \text { </xs:element > }
\end{aligned}
$$$$
\text { use="required" / }>
$$

\subsection{Mapping of simple data type}

The structure of a simple data type is simple, considering mapping it as simple data type or attributes in XML Schema. Due to in the EXPRESS language, an entity may contain more than one attribute value or element. Some attributes are closely related to the entities, such as the names and descriptive attributes in the entity. If the mapping is a simple type and can't reflect the close relationship with the entity, this attribute is mapped to the data type that is closely related to the entity attributes, while simplifying the structure at the same time better reflect the attributes and entities directly affiliated ${ }^{[6]}$.

In the real type, for example, the identifier in the EXPRESS language is directly mapped to the name attribute value of the XML Schema language simple type. The data type REAL mapping is to the base attribute value in the type restriction, and the Restrictions on the size of data in the WHERE domain rules map to the extent faces in the constraint surface. It is worth noting that, if there are constraints, REAL can only map to decimal and its precision maps to the precision face in the constraint surface.

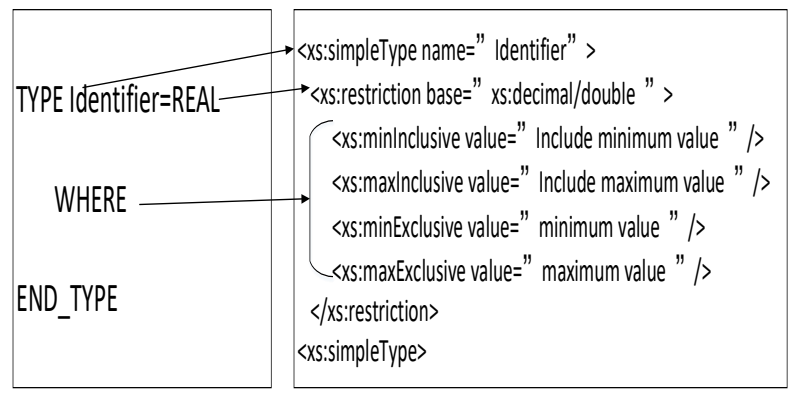

Figure 1. Real type mapping mechanism.

\subsection{Mapping of aggregate data type}

Aggregate Data Type Arrays, tables, and collections can be mapped to simple type of table type of XML Schema. The general aggregate type appears in the entity, consider mapping it to an entity's element. The identifier of the aggregate type is mapped to the name attribute value of the element, and the identifier of the constituent element is mapped to the name attribute value of the child element. SET [1 :?] indicates that the component has at least one max Occurs attribute mapped to XML Schema and the attribute value is unbounded.

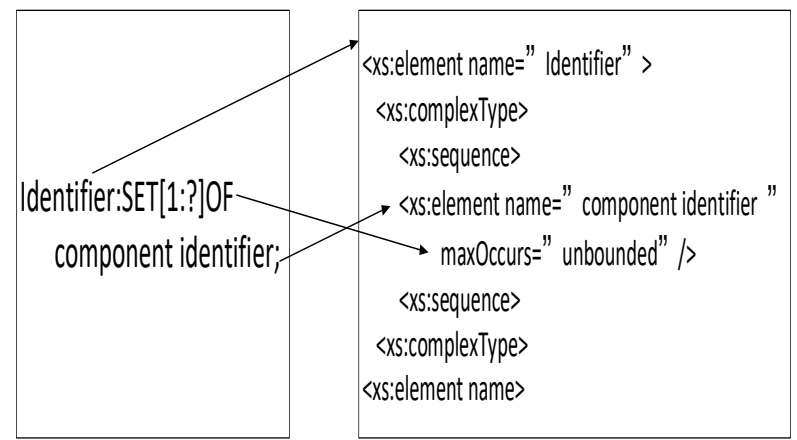

Figure 2. Aggregate data type mapping mechanism.

\subsection{Mapping of structural data type}

Structural data type in EXPRESS includes enumeration data type and selecting data type. The enumeration data type can be mapped to a qualified derived type from the simple type of XML Schema. The identifier is mapped to a simple type name attribute, and the base attribute in restriction needs to determine the data type according to the value. The content of the enumeration type is mapped to the enumeration surface in the constraint type constraint surface. 


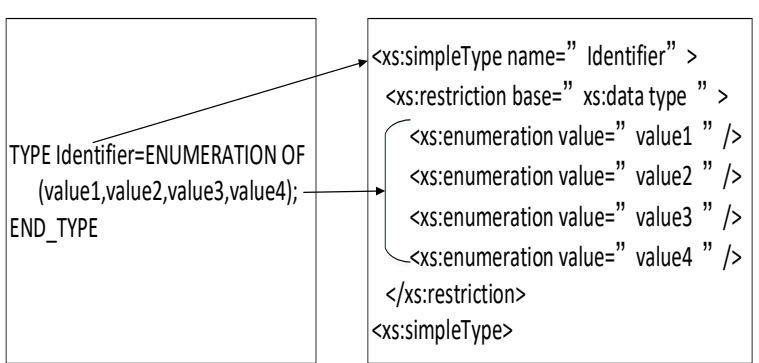

Figure 3. Enumeration data type mapping mechanism.

Due to XML Schema simple type can't directly describe the selection process, the selection type is mapped to a complex type in XML Schema, the selection type is mapped to the name attribute value in a complex type, and the only one type making a choice statement on complex type declarations, and choosing the type's concrete value mapping for the element attribute values in the choice declaration, it's worth noting that in the content model as specified, only one element can be typed in the instance document,value1, value2, value 3 choose a value, we can't insert the three values together.

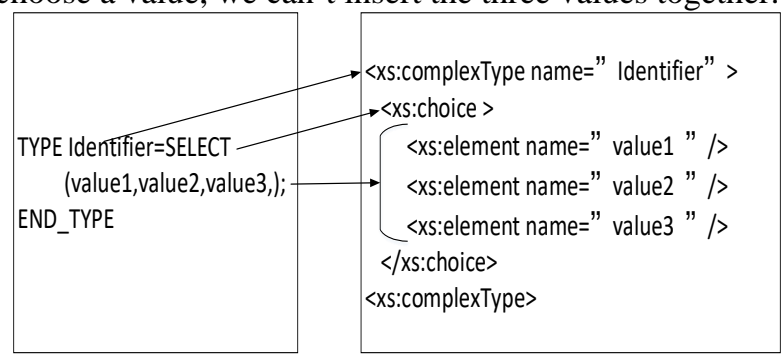

Figure 4. Select data type mapping mechanism.

\section{XML SCHEMA DESCRIPTION OF CEM DATA TYPE}

The common element model (CEM) is one of the most basic models in the AI-ESTATE standard (IEEE1232 standard). All other models are extended by CEM. The standard uses the EXPRESS language to describe the model. Due to the limitation of the EXPRESS language, Diagnostic knowledge is difficult to be shared and reuse, so consider to map EXPRESS language into XML language, make information with portable Io and interoperability.

\subsection{Analysis of data type in CEM}

The data type involved in CEM are simple data type and structural data type and entities. Based on the first two parts, this paper gives an example of how to standardize the entities. There are 9 simple data type in CEM, of which 6 are real type, namely Confidence Value, Cost Value, Failure Rate, Distribution Point, Probability Value, Time Value, and 3 string type, namely Description Type, Name Type and Qualifier Type respectively. Structural data type of 9, of which enumeration type 8, which are ActionCost Type, NonTime Unit, Outcome Values, ResourceCost Type,
Role, SeverityCategory, TimeBaseline, TimeUnit, select a data type, as FailureDistribution.

\subsection{XML Schema Description of CEM Data Type}

Mapping the EXPRESS data type that appear in CEM to XML Schema following the mapping rules in Section 2. Mapping real and string type to simple type or properties of XML Schema, those type that appear in the entity and are closely related to the entity are mapped as attributes of the entity, the rest are mapped as simple XML Schema type and where the domain rules are mapped as XML Schema constraints, it is worth noting that not every type has 12 constraint surfaces, each type may have one or more constraint surface.

Mapping the enumeration data type to simple XML Schema type using the restriction statement. Mapping selection data type to a complex type of XML Schema. Using choice declaration, and there can not have more than one element in an instance document.

The following string type Description Type, enumeration type ActionCost Type and selection type FailureDistribution as an example, its XML description.

(1)Enumeration type of XML Schema description

The enumeration type Color is described in the EXPRESS language as:

TYPE Color=ENUMERATION OF

(RED,

BLACK

WHITE,

BLUE,

GREEN,

YELLOW,

PINK);

END TYPE;

According to the mapping rules, using XML Schema described as:

<xs: simpleType>

$\langle x s$ : restriction base="XS: string">

$\langle x s$ : enumeration value=" RED"/>

$\langle x s$ : enumeration value=" BLACK"/>

$\langle x s$ : enumeration value=" WHITE"/>

$\langle x s$ : enumeration value=" GREEN"/>

$\langle x s$ : enumeration value=" YELLOW"/>

$\langle\mathrm{xs}$ : enumeration value=" $\mathrm{PINK} " /\rangle$

$</ \mathrm{xs}$ : restriction $>$

$</ x s$ : simpleType $>$

(2) XML Schema description of the string type

The EXPRESS language for string type NameType in CEM is described below:

TYPE NameType=STRING;

END_TYPE;

Mapping it as an XML Schema according to the mapping rules to simple type is built into a simple type string, which can be constrained in the constraint surface if the length of the string is limited, as described in the following XML Schema:

<xs: simpleType name=" NameType" >

$<\mathrm{xs}$ : restriction base $=$ " XS: string” / $>$ 
$</ x s$ : simpleType $>$

(3)Selection type of XML Schema description

Selection type of FailureDistribution EXPRESS described as under:

TYPE FailureDistribution=SELECT

(FailureRate, ProbabilityDistribution);

END_TYPE;

After mapping into XML Schema:

<xs: complexType name="FailureDistribution">

$<x s$ : choice>

$\langle x s$ : element nalne="FailureRate"/ $>$

$\langle$ xs: element name = "Probability Distribution "/>

$</ \mathrm{xs}$ : choice $>$

$</$ xs: complexType $>$

\subsection{XML Schema Description of CEM Entity}

Entities are more complex data type in EXPRESS and may include one or more of simple type, aggregate type, and structural type. CEM contains 25 entities. The mapping of entities is based on the mapping of simple type, aggregate type and structural type. The following takes the Outcome entity as an example to describe XML.

The entity Outcome is an abstract superclass of the entities diagnose_outcome and test_outcome. It contains three attributes and a UNIQUE rule; the three attributes name, description, and confidence refer to the definition type, where confidence is optional and the UNIQUE rule restricts the uniqueness of the name attribute, as follows:

ENTITY outcome

\section{ABSTRACT SUPERTYPE}

$\mathrm{OF}$

(ONEOF(diagnosis_outcome, test_outcome));

name : name_type;

description : description_type;

confidence : OPTIONAL confidence_value;

UNIQUE

one_name : name;

\section{END_ENTITY;}

Since the confidence attribute of the entity outcome is optional, mapping is achieved through attribute use="optional", and the name and description attributes need a use="required" constraint to map it to a complex type attribute.

The standard description of the entity outcome XML Schema is shown in Figure 5.

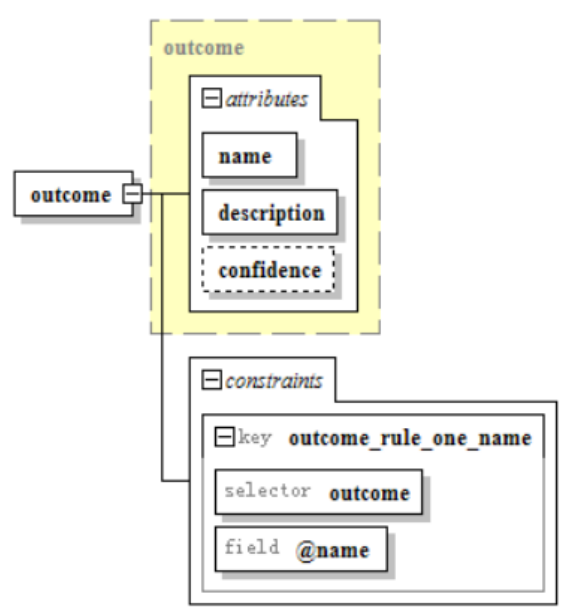

Figure 5. Outcome entity XML Schema description.

\section{CONCLUSION}

This paper starting from the data type of EXPRESS language and XML language, studies the mapping mechanism of simple data type, aggregate data type and structural data type to XML Schema data type in EXPRESS respectively. Based on the analysis of the data type of common element model in AI-ESTATE standard, this paper discusses how to describe the data type in common element model by using XML Schema and illustrates the mapping of entity type in the model, which is more convenient for diagnosis knowledge sharing and porting also provide the foundation for the XML Schema description of other diagnostic models in the standard.

\section{REFERENCES}

1. Tong Jiang, Cai Yuanwen, Xing Xiaochen. The Application of next generation ATS in The Domestic Aerospace Test System Architecture [J]. Aerospace Control, 2011,29(2): 75-80.

2. IEEE. IEEE Std 1232-2010, IEEE Standard for Artificial Intelligence Exchange and Service Tie to All Test Environments [S].

3. IEEE. IEEE Trial--Use Standard for Artificial Intelligence Exchange and Service Tie to A1I Test Environments(AI-ESTATE) : Data and Knowledge Specification. IEEE Std 1232 . 11997 [S].

4. Santiago Delgago, Anand Jain . ATML as a framework for reducing development time and maintenance of next generation test system[J]. IEEE 2007: 174-177.

5. IEEE . IS010303-28:2007. Industrial automation systems and integration-Product data representation and exchange-Part 28 : Implementation methods: XML representations of EXPRESS schemas and data . using XML schemas[z]. 
6. Barkmeyer E J, Lubell J. XML Representation of EXPRESS models and data[EB/OL . http : //www . mel. nist. gov/msidlibrary/doc/xse2001 . pdf . 2001 . Information on http://www.weld.labs.gov.cn 